\title{
FLIGHT MANAGEMENT SYSTEM EXECUTION OF IDLE-THRUST DESCENTS IN OPERATIONS
}

\author{
Laurel Stell, NASA Ames Research Center, Moffett Field, CA
}

\begin{abstract}
To enable arriving aircraft to fly optimized descents computed by the flight management system (FMS) in congested airspace, ground automation must accurately predict descent trajectories. To support development of the trajectory predictor and its error models, commercial flights executed idle-thrust descents, and the recorded data includes the target speed profile and FMS intent trajectories. The FMS computes the intended descent path assuming idle thrust after top of descent (TOD), and any intervention by the controllers that alters the FMS execution of the descent is recorded so that such flights are discarded from the analysis. The horizontal flight path, cruise and meter fix altitudes, and actual TOD location are extracted from the radar data. Using more than 60 descents in Boeing 777 aircraft, the actual speeds are compared to the intended descent speed profile. In addition, three aspects of the accuracy of the FMS intent trajectory are analyzed: the meter fix crossing time, the TOD location, and the altitude at the meter fix. The actual TOD location is within $5 \mathrm{nmi}$ of the intent location for over $95 \%$ of the descents. Roughly $90 \%$ of the time, the airspeed is within 0.01 of the target Mach number and within 10 KCAS of the target descent CAS, but the meter fix crossing time is only within $50 \mathrm{sec}$ of the time computed by the FMS. Overall, the aircraft seem to be executing the descents as intended by the designers of the onboard automation.
\end{abstract}

\section{Introduction}

In congested airspace today, controllers direct aircraft to descend with a vertical profile resembling stairsteps. Since air density, and hence drag, increase as the aircraft descends, significant reductions in fuel consumption and emissions would result if aircraft stayed at cruise altitude longer and then descended smoothly at idle thrust. The flight management system (FMS) on a large jet can compute the location of top of descent (TOD) assuming an idle-thrust descent. To merge aircraft, however, controllers impose level flight segments, which make it much easier for them to estimate the relative speeds of two aircraft given their calibrated airspeeds (CAS). The ultimate goal of the research described in this paper is to enable more fuelefficient descents in congested airspace. This requires development of a trajectory predictor as well as its error models so that aircraft can be given clearances with a low probability that a revision will be needed later. The along-track error must clearly be small enough to ensure lateral separation. Accurate prediction of the vertical profile is also essential to ensure vertical separation from aircraft at different altitudes, including crossing traffic. In the future, datalink will give controllers and ground systems access to the FMS trajectory prediction, but a ground predictor will still be necessary to create "what-if" trajectories in the cases where preferred trajectories would be likely to result in conflict.

Development of the predictor and operational concept requires knowledge of the prediction error. Causes of this error can be categorized as

- error in the current aircraft state used as an initial condition by the predictor,

- differences between the models used by the FMS and by the ground automation predictor (which seem to be primarily differences in the aircraft thrust and drag models and in the meteorological conditions), and

- error in the execution of the descent procedure.

This paper investigates errors in the first and last categories using operational data.

Commercial flights have been executing the Oceanic Tailored Arrival (OTA) procedure for over three years at San Francisco International Airport (SFO). This paper shows results of analyzing over 50 of these descents, which is a superset of the 11 analyzed in Coppenbarger, Mead, and Sweet [1]. This set of descents has two advantages over the operational data from Denver analyzed by Stell [2]:

- the flights are spread out temporally rather than occuring in a three-week period and

- Automatic Dependent Surveillance-Contract (ADS-C) messages are recorded for these flights, which provides not only state data but also intent data. 
Radar data are also archived, which means the positions and altitudes from two sources of state data can be compared. The sample size is much too small and the analysis too limited to assess the accuracy of the radar system. (For that, see Paglione and Ryan [3].) Instead, this comparison is intended as a sanity check and to give insight into the subsequent analysis of the execution of the descents. The ADS-C state data also include the airspeed, which is compared to the target speed profile. This augments the analysis by Stell [4] of the speed profile in descents executed in a test bench simulator with a commercial FMS. Finally, three aspects of the accuracy of the FMS intent trajectory are analyzed: the meter fix crossing time, the TOD location, and the altitude at the meter fix. Klooster, Del Amo, and Manzi [5] also analyze the accuracy of the FMS-computed times, but in their procedure the autopilot controls the time of arrival. In the OTA procedure, the autopilot tries to maintain the specified speed profile, so the intent time is a prediction that will reflect not only execution errors but also errors in the wind forecast.

\section{Operational Procedures and Data}

This section describes the operational procedures and data. Coppenbarger, Mead, and Sweet [1] give more information about the procedures. At the Oakland Air Route Traffic Control Center (ARTCC), controllers use the Advanced Technologies and Oceanic Procedures (ATOP) system for communication with and surveillance of aircraft in oceanic airspace. ATOP provides two-way digital messaging between the ARTCC and the flight deck through Controller-Pilot Datalink Communications (CPDLC). When using the OTA procedure, the route clearance, which consists of lateral waypoints with any speed or altitude restrictions, is uplinked by the ARTCC to the aircraft and then directly loaded into the FMS through its Future Aircraft Navigation System (FANS) avionics interface.

The CPDLC messages are archived, which provides the intended speed profile for this analysis. The radar tracks are also archived, from which the cruise altitude and horizontal trajectory are extracted. The Terminal Radar Approach Control Facility (TRACON) radar data are used whenever they are available because the update frequency is about $(5 \mathrm{sec})^{-1}$. This radar does not cover the entire descent, however, so the ARTCC radar data, which have a frequency of about $(12 \mathrm{sec})^{-1}$, are also used. Finally, ADS-C messages are archived, providing both state data (Basic Group) and FMS intent (Intermediate Projected Intent Groups (IPIG)). Only one FMS intent trajectory is used for each descent, and it is from the last ADS-C message in which the altitude is still at cruise. The trajectory change points in this message include both the FMScomputed TOD and the meter fix. About half the ADS-C messages are $64 \mathrm{sec}$ apart as specified by the contract; another $40 \%$ are $120 \mathrm{sec}$ apart; and almost all the remaining 10\% are 1000-3000 sec apart due to various technical glitches. Any controller intervention is also recorded, and those flights are excluded from this analysis.

The analysis in this paper considers only the initial descent down to the location of the first altitude and speed constraint. The FMS typically uses the constraints shown in Figure 1 to build its intended trajectory, although the descents analyzed here include variations that will be described below. The parameters shown in each box in Figure 1 are specified for that segment or that point of the descent. The first segment after TOD has constant Mach number, which is the same as the cruise Mach number. As the altitude decreases, the CAS increases until it reaches the target descent CAS specified by the controller. The next segment is then flown at that CAS. Finally, the aircraft pitches up to decelerate to the speed constraint at the meter fix. The analysis in this paper ends at the end of the first deceleration segment in the descent.

The OTA procedure was first used in the following test periods:

- August 17 - September 6, 2006

- December 13 - 23, 2006

- January 2- 9, 2007.

For these flights, the first constraints were $11,000 \mathrm{ft}$ and $240 \mathrm{kt} \mathrm{CAS} \mathrm{(KCAS)} \mathrm{at} \mathrm{the} \mathrm{meter} \mathrm{fix.} \mathrm{The} \mathrm{test} \mathrm{in-}$ volved commercial flights from Honolulu to San Francisco, operated by a single airline and scheduled at the same time each day. The flight was chosen because the aircraft was a Boeing 777 equipped with FANS and because its scheduled arrival time was 5:30 a.m. local when there is very little traffic. Of the test flights, 20 are suitable for this analysis.

Use of the OTA procedure on a regular basis began December 3, 2007. The data used in this analysis are available for two participating airlines. The available route clearances were tweaked several times in the first two months, so this analysis only uses flights after January 25, 2008. Until September 8, 2008, the first altitude and speed constraints were not actually part of the route clearance but were instead the common speed constraint of $240 \mathrm{KCAS}$ at $10,000 \mathrm{ft}$. The location of 


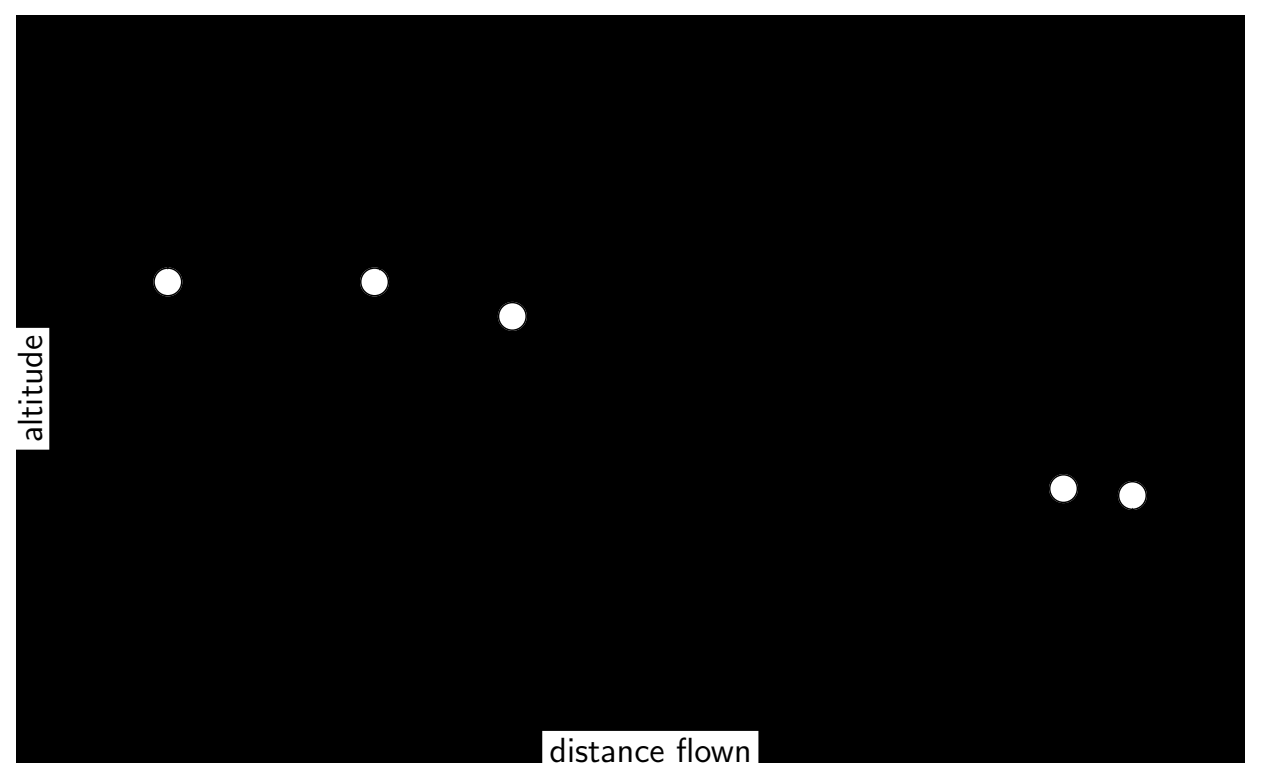

Figure 1. Idle-thrust Descent Schematic

this point in the FMS intent trajectory is used in the same way as the meter fix location was used for the earlier flights. For convenience, it will be referred to as the meter fix. From this time period, 28 descents are included in this analysis.

After September 8, 2008, a speed constraint of $250 \mathrm{KCAS}$ was added at the waypoint PIRAT. An altitude constraint of "at or below 15,000 ft" was also added there. The appropriate ADS-C IPIG gives the intended altitude at that location. At the end of November 2008, archiving of the radar track data was suspended for several months, so that is the extent of the data used for this preliminary analysis. From this time period, only three descents are acceptable for this analysis.

This means a total of 51 flights are available for this analysis, but some of them are missing one or more data sources, which means none of the plots in this paper include all 51 descents. All of the descents analyzed in this paper are B777-200 aircraft. In 2008, some FANS-equipped B747-400 aircraft arriving at SFO from oceanic airspace started participating in the OTA procedure, but too few of them had all the data necessary for this analysis.

To simplify visualization of the data, the horizontal location is specified as along-track path distance relative to the meter fix. The horizontal flight path is chosen by looking for one of the predefined routes close to the radar track. To complete the description of the data extracted from the radar data, the TOD location is chosen to be the point where the aircraft left cruise altitude.

\section{Comparison of State Data}

Both the radar data and ADS-C state data include the position and altitude of the aircraft. This section shows comparisons of these two data sources and discusses the differences. Of the 51 flights, 47 have both ADS-C state data and radar data. Of these, seven of the flights in 2008 have only ARTCC radar data - no TRACON radar data. For the field trials, the TRACON radar data consistently start 25-30 nmi from the meter fix. For decents in 2008 before September 8, the TRACON radar data start 28-33 nmi from the meter fix, except for one descent in which they start $15 \mathrm{nmi}$ from the fix. For descents after September 8, 2008, the TRACON radar data start $15-20 \mathrm{nmi}$ from the fix. These changes are due to the fact that, as described above, the location referred to as the meter fix in this paper moved when procedures changed.

\section{Comparison of Horizontal Position}

The first analysis compares the horizontal position of the aircraft given by radar data to that given by ADS-C state data. The crosstrack distance between the ARTCC radar and ADS-C tracks is generally less than 
$0.5 \mathrm{nmi}$; whereas the crosstrack distance between the TRACON radar and ADS-C tracks is less than $1 \mathrm{nmi}$, although this difference is $1-3 \mathrm{nmi}$ for the flights in August-September 2006. The reason the crosstrack distance is smaller for ARTCC radar data than for TRACON data is probably that the ARTCC radar system coordinate mapping happens to be more accurate in the geographic area considered here.

More important for advising continuous descents is the along-track difference, which would affect intrail separation. The upper plot in Figure 2 shows the difference in path distance as a function of time. Since there is rarely a radar report with exactly the same timestamp as an ADS-C message, this plot is based on linear interpolation. Suppose there is an ADS-C message at time $t$ with path distance $d_{a}(t)$. The path distance $d_{r}(t)$ from radar data is obtained by linear interpolation. The value $\left[d_{a}(t)-d_{r}(t)\right]$ is then indicated by the vertical coordinate of a marker in the plot. Since the times span over two years, the horizontal coordinates are shifted so that zero indicates the time at which the aircraft crossed the meter fix. Each line in the plot corresponds to one descent. The lower plot in Figure 2 is created in the same way but shows the difference in time as a function of path distance. In general, radar data within $300 \mathrm{sec}$ or $40 \mathrm{nmi}$ of the meter fix are from the TRACON radar, except in those descents that had no TRACON radar data.

The descents with small differences in the TRACON coverage area are the set of flights in December 2006 and January 2007, which are ignored for the moment. Plots like those in Figure 2 but showing only ARTCC or only TRACON radar data show that the times of the radar hits are 5-15 sec late for the ARTCC radar and 5-13 sec late for the TRACON radar. These differences are due to the fact that NASA receives radar data without timestamps. The timestamps are added by the application that processes and archives them at NASA. The time differences suggest an overhead delay of $5 \mathrm{sec}$ (which corresponds to about $0.6 \mathrm{nmi}$ in the upper plot) for processing by the Host radar, transmission to the FAA's William J. Hughes Technical Center, transmission back to NASA Ames Research Center, and waiting to be processed by the NASA application. The variation in the differences is most likely due to the fact that different aircraft are painted at different times in a single radar sweep. This would suggest a range of $12 \mathrm{sec}$ in the differences in the ARTCC radar times and a range of $5 \mathrm{sec}$ in the differences in the TRACON radar times, which is reasonably close to the observed ranges of $10 \mathrm{sec}$ and $8 \mathrm{sec}$, respectively. The reason for essentially no delay or variation in the differences for the TRACON radar data in December 2006 and January 2007 is unknown.

To maximize the use of fuel-efficient descents in congested airspace, the meter fix crossing time must be predicted accurately enough. A common estimate of this accuracy requirement is absolute error less than $30 \mathrm{sec}$ (see [6], for example). The preceding results suggest that, in order to achieve this, the predictor input will need to have timestamps consistent with the ground truth timestamps. This also means that using downlinked predictions may actually cause some problems if other system components are using radar data that do not have radar paint times.

\section{Comparison of Altitude}

The current altitude in the ADS-C messages is the same as the altitude transmitted by the Mode-C transponder and used by radar. These are pressure altitudes, which means they are based on a reference pressure of $29.92 \mathrm{in} \mathrm{Hg}$ even below the transition altitude FL180. If the altitude is above FL180, then the Host computer transmits it unchanged in the radar data. If the altitude is below FL180, however, the Host computer uses the current QNH altimeter setting ${ }^{1}$ to convert the altitude to barometric altitude before transmitting the data. Therefore, a direct comparison of the ADS-C altitudes with the radar altitudes gives differences as large as $600 \mathrm{ft}$ as shown in the upper plot of Figure 3. To correctly compare the ADS-C state altitudes with the radar altitudes, the radar altitudes less than $18,000 \mathrm{ft}$ must be converted back to pressure altitudes.

The equation used to relate altitude $h$ in feet to pressure $P$ is

$$
h=\alpha\left[1-\left(\frac{P}{P_{\mathrm{ref}}}\right)^{\beta}\right],
$$

where $\alpha=145,447 \mathrm{ft}, \beta=0.190255$, and $P_{\text {ref }}$ is a reference pressure. To convert $h_{1}$ based on reference pressure $P_{1}$ to $h_{2}$ based on reference pressure $P_{2}$, use

$$
h_{2}=\alpha\left[1-\left(1-\frac{h_{1}}{\alpha}\right)\left(\frac{P_{1}}{P_{2}}\right)^{\beta}\right] .
$$

\footnotetext{
${ }^{1}$ An altimeter that is on the runway and set to the QNH altimeter setting will show the published runway altitude.
} 

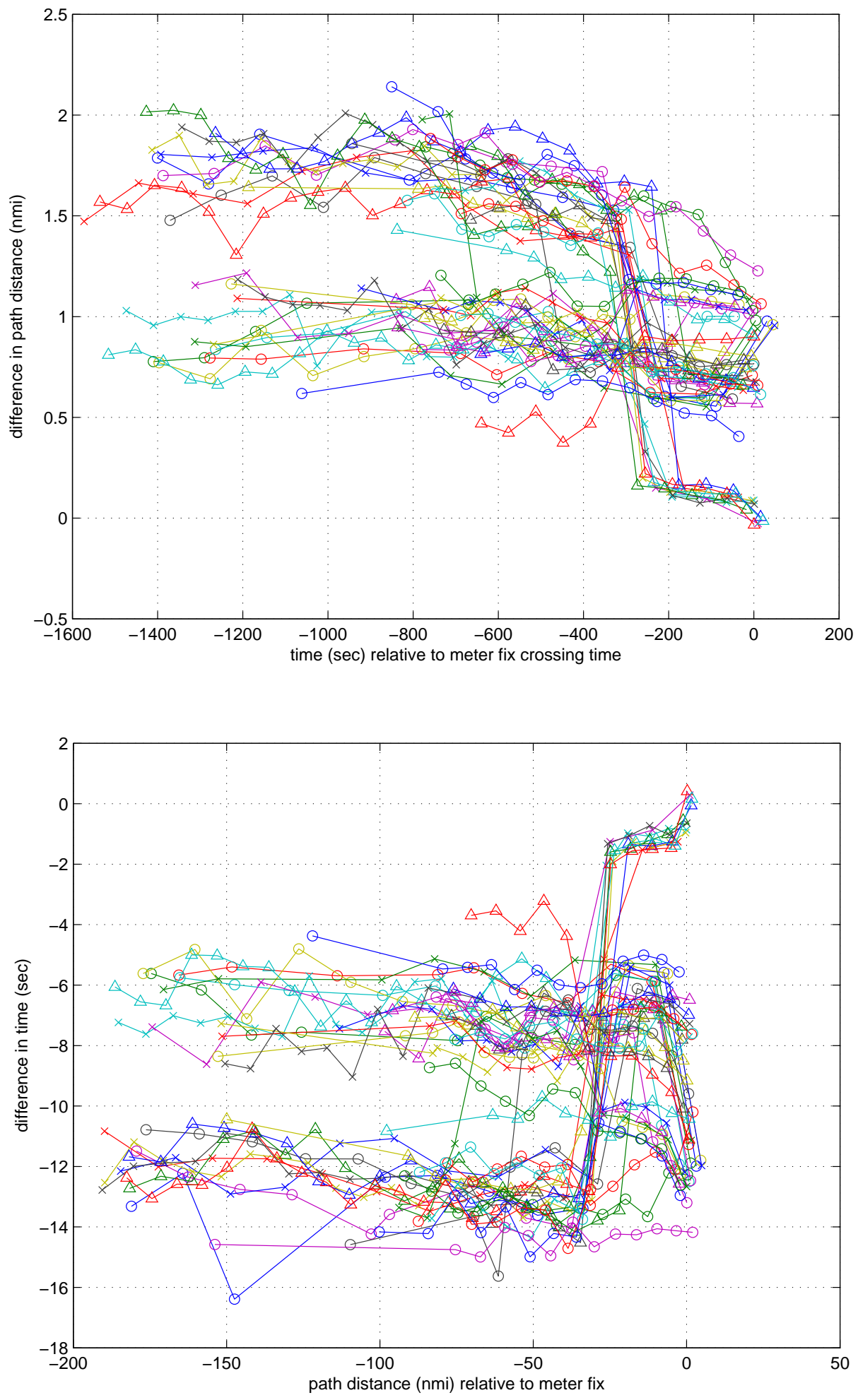

Figure 2. Comparison of Along-track Distance 

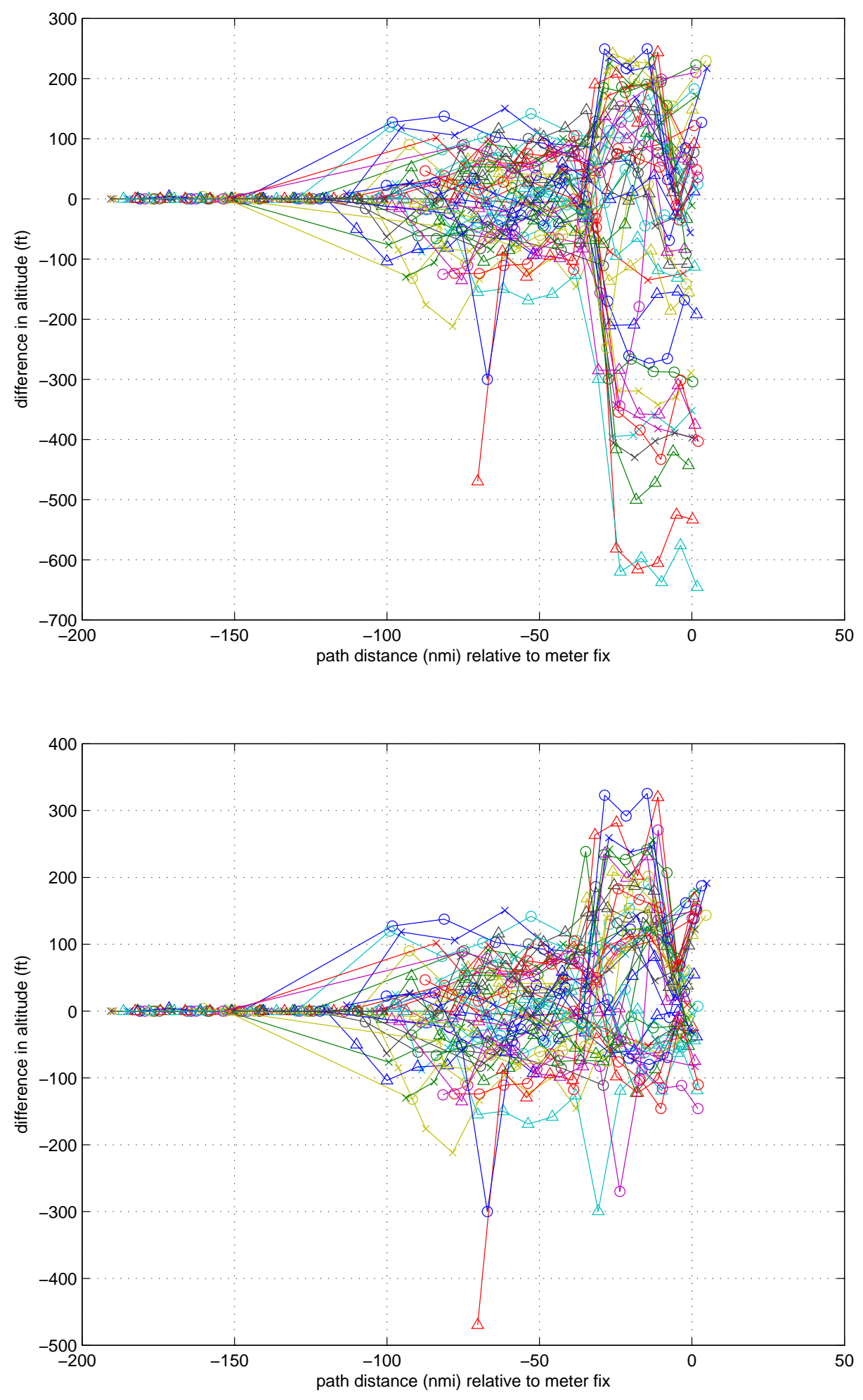

Figure 3. Comparison of Altitude 


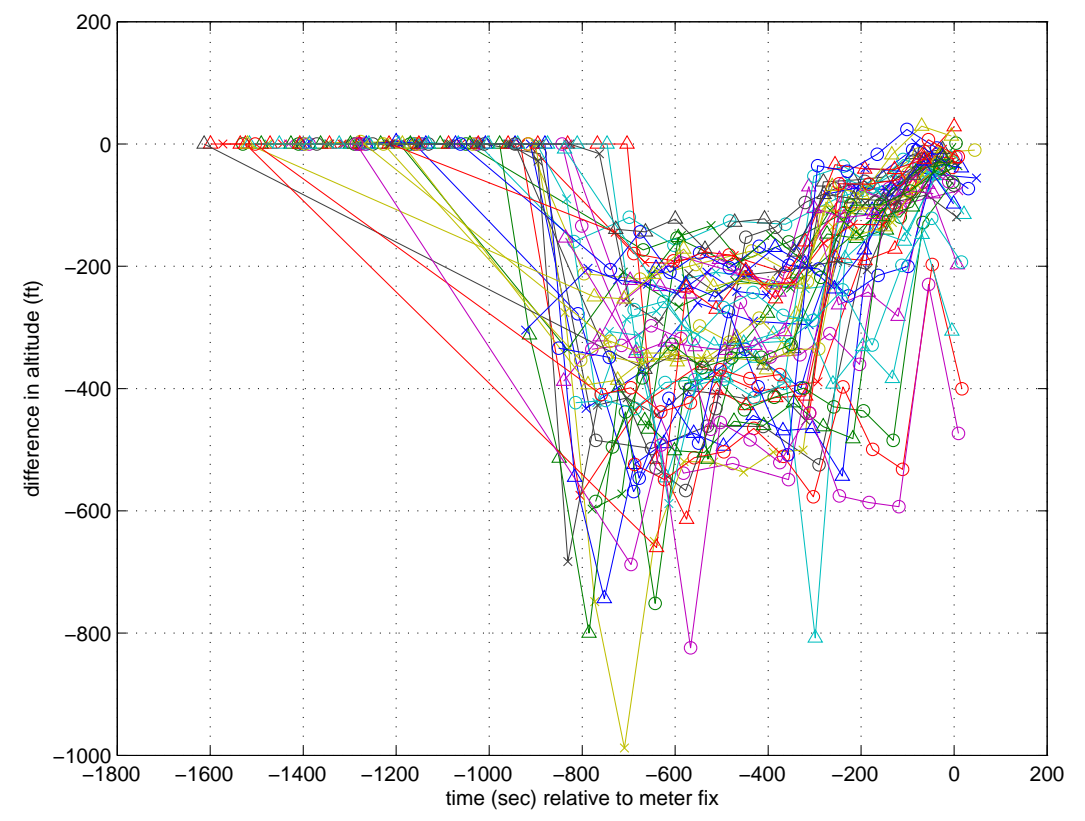

Figure 4. Comparison of Altitude as a Function of Time

The historic QNH altimeter settings were obtained from the National Climatic Data Center (NCDC) online archive. The lower plot in Figure 3 shows the differences between the ADS-C altitudes and the adjusted radar altitudes obtained from equation (2) with $P_{1}$ equal to the $\mathrm{QNH}$ altimeter setting and $P_{2}$ equal to $29.92 \mathrm{in} \mathrm{Hg}$. These differences are shown as a function of path distance so that the delay in the radar timestamps is irrelevant.

When the aircraft is in cruise, there is essentially no difference between these two altitude measurements. While the aircraft is above FL180, the absolute value of the difference is almost always less than $200 \mathrm{ft}$ and is less than $100 \mathrm{ft}$ for about $80 \%$ of the measurements. After the aircraft descends below FL180 roughly $40 \mathrm{nmi}$ before the meter fix (but the exact distance varies between descents), the absolute differences are less than $200 \mathrm{ft}$ for over $95 \%$ of the measurements.

These results indicate that it does not matter whether the predictor uses for its initial condition the cruise altitude from an ADS-C message or from radar. If there are altitude constraints below FL180, however, the predictor must take into account the QNH altimeter setting rather than simply subtracting from cruise altitude to determine the change in altitude in the descent.
Figure 4 shows the same altitude differences as in the lower plot of Figure 3 but as a function of time instead of path distance. These differences based on the data timestamps are much larger and are more heavily biased than the differences based on path distance. This further supports the conclusion that the differences shown in Figure 2 are primarily due to incorrect timestamps rather than bias in the radar positions.

\section{Comparison of Actual Descent to In- tended Descent}

The difference between the actual descent and the FMS trajectory prediction would clearly be important in the future when the intent trajectory is available to controllers and ground systems via datalink. Even for more near-term goals, however, these differences give insight into the predictability of the descents by ground automation systems. This is particularly true for comparison of the actual and intended airspeeds, since this does not depend upon the FMS prediction algorithm, and this comparison is in the first subsection. Ideally, the along-track error and the error in the vertical profile of the FMS intent trajectory would also be investigated. Unfortunately, the IPIGs for the descents used here only include two trajectory change points between 
TOD and the meter fix, which is not enough to assess the errors for the entire descents. Instead, the errors in the meter fix crossing time are shown as an indication of the along-track error. In the procedure used in these descents, the autopilot is not directly attempting to meet its predicted meter fix crossing time but is instead trying to maintain the target speed profile. The error in the altitude at the meter fix location is discussed next. The errors in the TOD location are shown in the last subsection to confirm that the autopilot will usually start the descent near the location computed by the FMS. When comparing the actual trajectory to the intent trajectory, the FMS prediction used is the one from the last ADS-C message in which the current altitude is still at cruise.

\section{Execution of Speed Profile}

The ADS-C state data include airspeed given as Mach number. This makes it easy to compare the actual and intended airspeeds in cruise and in the constant Mach segment, although the extent of the constant Mach segment must be estimated. The transition from the constant Mach segment to the constant CAS segment is approximated from the ADS-C state data by essentially choosing the point where the target descent CAS is captured and the Mach number starts decreasing, although there are some additional conditions to handle the noise in the reported Mach numbers. The upper plot in Figure 5 shows the difference between the actual and intended Mach numbers in cruise and in the constant Mach segment, with a positive difference meaning the aircraft is faster than intended. The horizontal axis is path distance relative to actual TOD in order to make comparison between descents easier. The TOD location used is the one extracted from the radar data because the much higher frequency of the radar data means it is more accurate than the one extracted from the ADS-C state data. This means the points in the plot with negative $x$ values are in cruise, whereas the points with positive $x$ values are in the constant Mach segment.

Comparison of the actual and intended airspeeds in the constant CAS segment requires converting the Mach number to KCAS. The equation for this is

$$
v_{c}^{2}=5 a_{0}^{2}\left\{\left(\frac{P}{P_{0}}\left[\left(\frac{M^{2}}{5}+1\right)^{\frac{7}{2}}-1\right]+1\right)^{\frac{2}{7}}-1\right\}
$$

where $v_{c} \quad$ airspeed in KCAS

$M \quad$ Mach number

$a_{0} \quad$ speed of sound at sea level

$P_{0} \quad$ pressure at sea level

$P$ pressure at current altitude

and the adiabatic index is assumed to be 1.4. The Standard Atmosphere model is used for $a_{0}$ and $P_{0}$, as well as to estimate the pressure as a function of the pressure altitude given in the ADS-C state data. The lower plot in Figure 5 shows the difference between the actual CAS and the target CAS in the constant CAS segment, with a positive difference meaning the aircraft is faster than intended. If the airspeed drops too far below the target airspeed, the autopilot engages the throttle. The plot suggests that this threshold is $10 \mathrm{KCAS}$ for the B777-200 FMS - and also suggests that the conversion of Mach number to CAS is reasonably accurate. On the other hand, if the airspeed is too fast, the autopilot does not directly attempt to control the speed but instead displays a message to the pilot to slow the aircraft. As a result, some of the aircraft keep increasing CAS throughout the constant CAS segment. Figure 5 indicates the aircraft are flying close to the intended speed profile in these continous descents, with errors within the bounds of the autopilot control algorithms. Figure 6 shows this in a different way, plotting the empirical distribution functions of the differences between actual and intended airspeeds at selected path distances. As in Figure 5, the distances in the upper plot are relative to the actual TOD, and the distances in the lower plot are relative to the meter fix. Changing the Mach number by 0.01 changes the true airspeed by about $5 \mathrm{kt}$ for the conditions in cruise and constant Mach segment in these descents. A 10 KCAS change in the constant CAS segment changes the true airspeed by roughly $15 \mathrm{kt}$. Rough calculations indicate that such a change in true airspeed could change the time between TOD and meter fix by $20-35 \mathrm{sec}$.

\section{Comparison of Meter Fix Crossing Time}

Comparison of the meter fix crossing time predicted by the FMS to the actual time requires extracting the actual time from the data. Extracting from the radar data will include the delays of up to $15 \mathrm{sec}$ in their timestamps discussed above. On the other hand, using the time of the ADS-C message with location closest to the meter fix will include error caused by the time between ADS-C messages. While the absolute value of this error is nominally less than $32 \mathrm{sec}$, it may be much larger due to missing ADS-C messages. Figure 7 

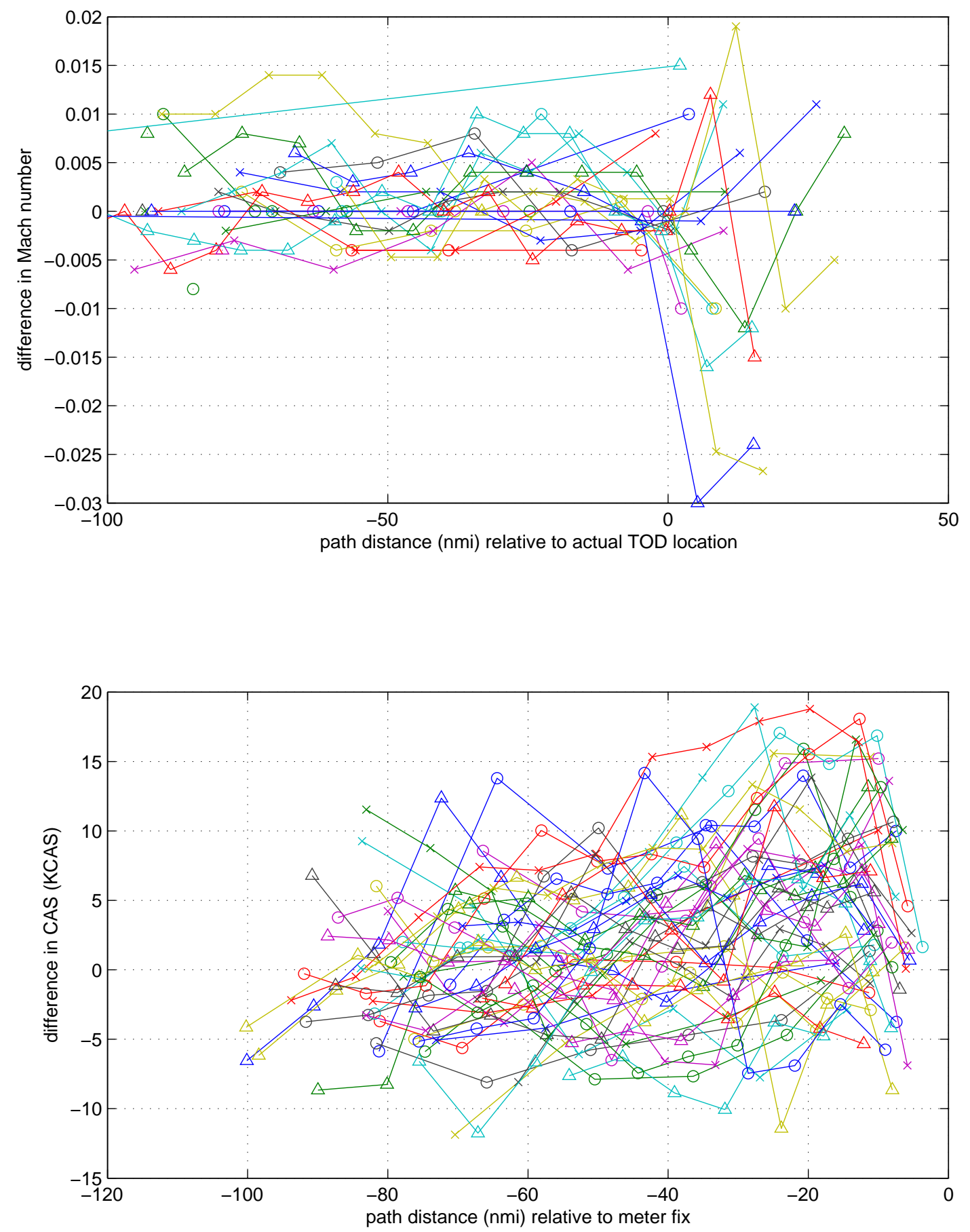

Figure 5. Comparison of Actual and Intended Airspeed 

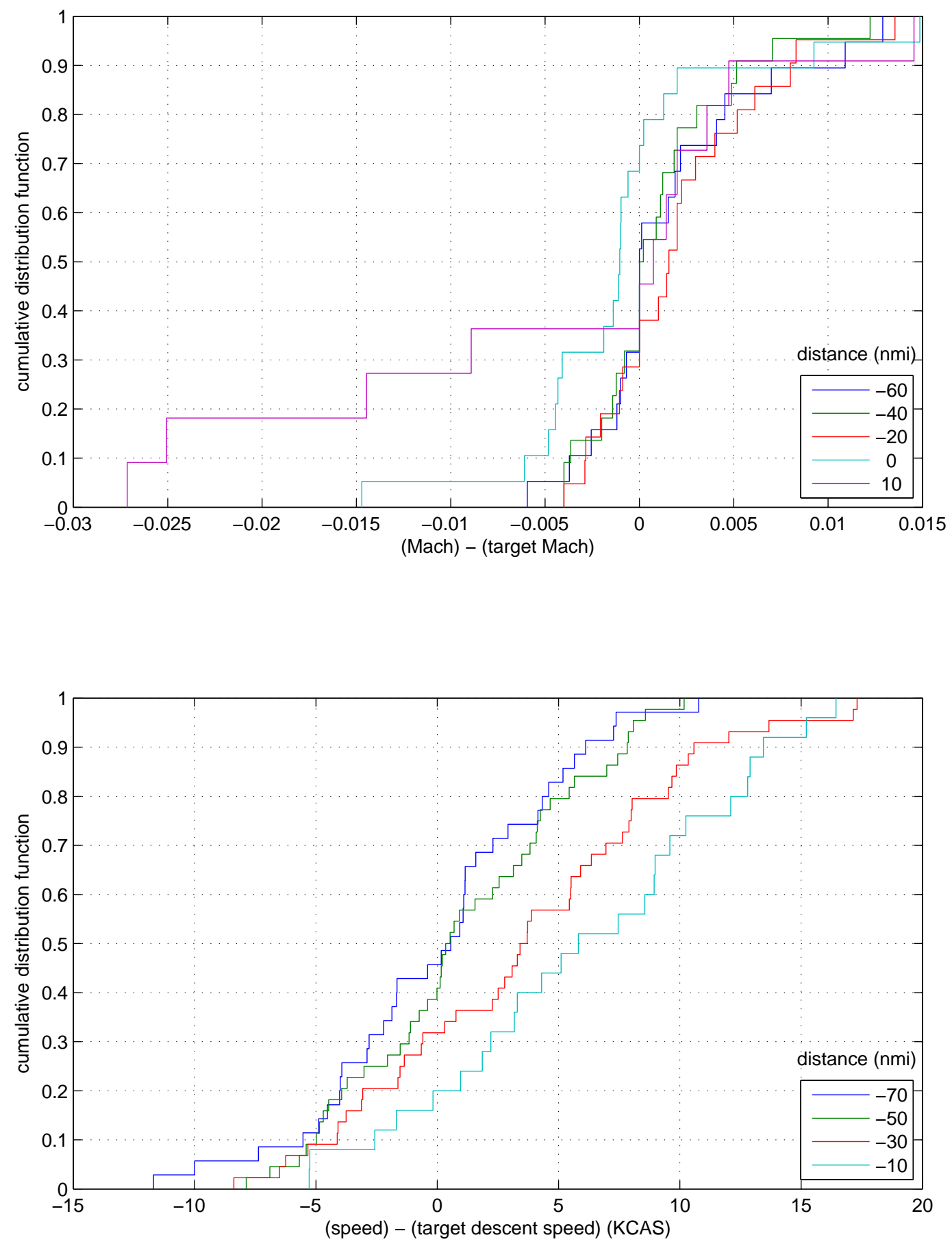

Figure 6. Distributions of Differences Between Actual and Intended Airspeed 


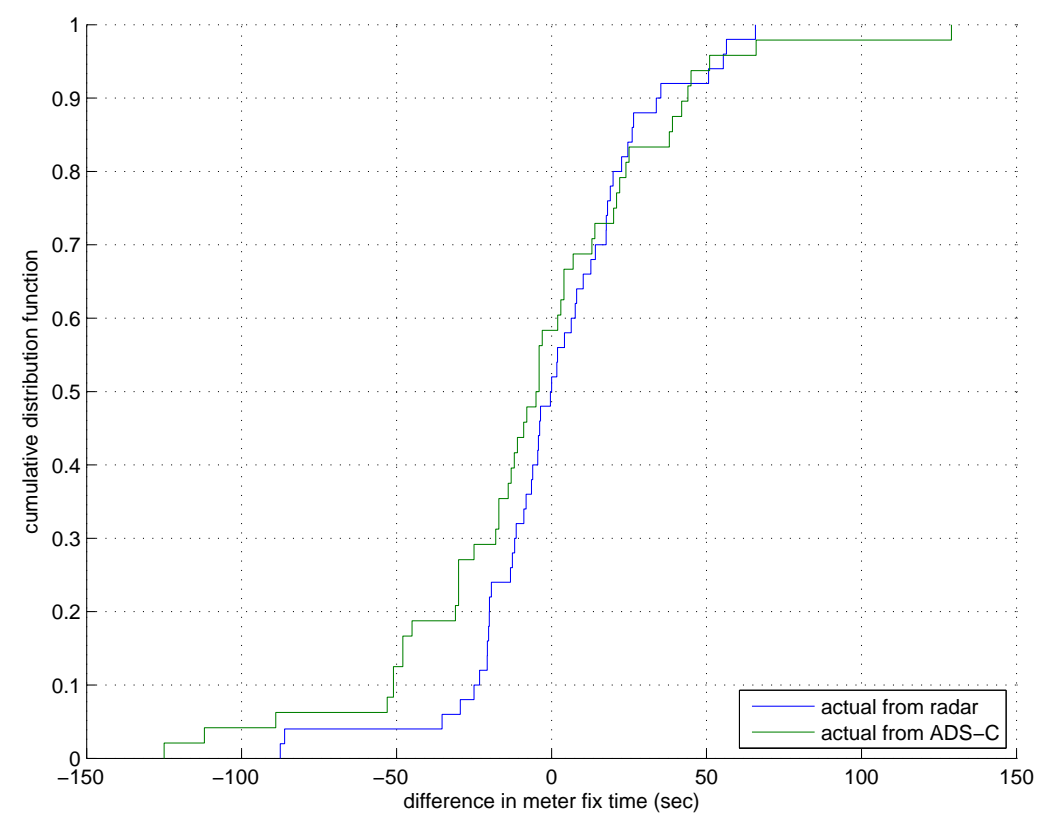

Figure 7. Comparison of Actual and FMS-predicted Meter Fix Crossing Time

shows the empirical cumulative distribution functions of the errors in the FMS prediction of meter fix crossing time, comparing both sources of actual data. Positive errors mean the FMS prediction is earlier than the actual time.

This should give a distribution based on the radar data that is skewed to the right; but no such effect is obvious, probably because the errors in the timestamps are relatively small compared to the prediction errors. The distribution based on the ADS-C state data has much larger tails, both positive and negative. The correlation coefficient is -0.7120 between these errors and the along-track distance (relative to the meter fix) of the location in the ADS-C message used for the actual time. Furthermore, the slope of the least squares linear fit to these points corresponds to a ground speed of about $285 \mathrm{kt}$, which is close to 240-250 KCAS at $10,000-11,000 \mathrm{ft}$ with no wind. Linear interpolation could be used to improve the estimate based on ADS-C state data of the actual meter fix crossing time. This would probably still not be a significantly better approximation of the errors than the distribution in Figure 7 based on the radar data. In summary, the error in the FMS prediction of the meter fix crossing time seems to be roughly $\pm 50 \mathrm{sec}$.

This seems consistent with the rough approximation in the previous subsection that execution error in the speed profile could cause fix time errors of 20 $35 \mathrm{sec}$ since there are also other sources of these errors.
These include errors in the wind forecast and errors in the vertical profile, which result in errors in calculation of true airspeed from CAS. Unfortunately, this 50-sec error falls short of the estimated accuracy requirement of $30 \mathrm{sec}$.

\section{Comparison of Meter Fix Altitude}

Figure 8 shows the errors in the altitude at the meter fix in the FMS intent trajectory. The actual altitude is extracted from radar data because they have much higher frequency than the ADS-C data and because they give barometric altitude - as does the FMS intent trajectory. A positive difference means the actual altitude is higher than intended. The flights between January 25 and September 8, 2008, have much larger errors than do the other flights. In this time period, the location where the FMS intent trajectory crossed $10,000 \mathrm{ft}$ is considered to be the meter fix for this analysis, and the intent trajectory used is the one in the last ADS-C message before TOD. After TOD, the FMS could change the location where it intended to cross $10,000 \mathrm{ft}$; and in seven of the descents, it moved the location more than $1 \mathrm{nmi}$. For the flights after September 8,2008 , the location used as the meter fix in this analysis is a fixed waypoint, but the altitude constraint is to cross at or below $15,000 \mathrm{ft}$. Consequently, the FMS could change its intended altitude at that waypoint during the descent. Unfortunately, only three 


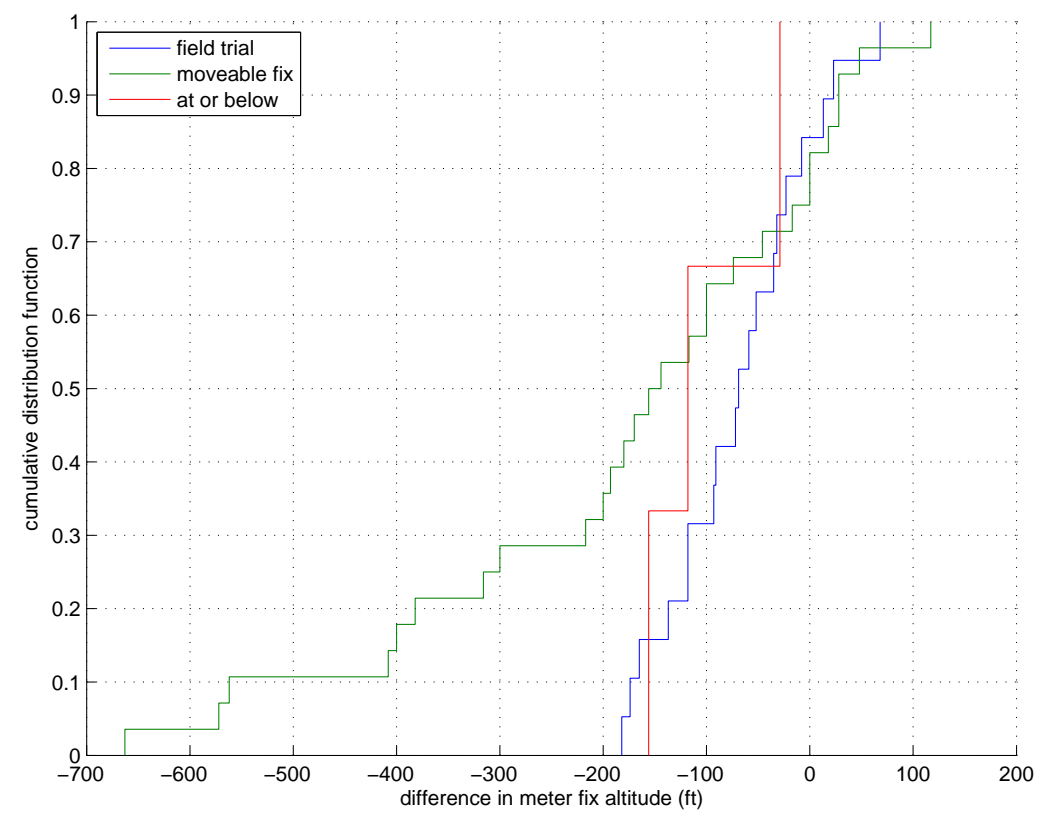

Figure 8. Comparison of Actual and FMS-predicted Altitude at Meter Fix

descents in this time interval have the data necessary for this comparison, which is much too small to draw any conclusions. Clearly, though, the choice of trajectory constraints can significantly impact the predictability of the trajectories, even if the intent trajectory is available to controllers and ground systems via datalink.

\section{Comparison of TOD Location}

Figure 9 shows the errors in the TOD location in the FMS intent trajectory. Extracting the location from the radar data has no known drawbacks, whereas extracting it from the ADS-C state data is inaccurate due to the low frequency of the ADS-C messages. Consequently, the plot only shows the comparison to actual location based on radar data. A positive difference means the actual TOD is closer to the meter fix than is the intent TOD. This is the case for only two of these descents because the autopilot initiates descent earlier than computed by the FMS in order to compensate for the FMS assumption of instantaneous changes in thrust and in vertical speed at TOD. The actual TOD location is $0-4 \mathrm{nmi}$ from the intent TOD location in $85 \%$ of the descents. This likely represents unavoidable variability in execution and is probably better than any ground predictor will be able to achieve.

\section{Conclusions}

Comparison of the position and altitude from radar data and from ADS-C state data highlights the importance of two issues:

- All components of a system should use timestamps that are consistent.

- The reference pressure for all altitudes must be clear and appropriate.

These issues also constrain the accuracy of the subsequent analysis of the accuracy of the FMS intent trajectory.

Overall, the aircraft seem to be executing the descents as intended by the designers of the onboard automation. The airspeed is generally within 0.01 of the target Mach number and within 10 KCAS of the target descent CAS. Despite this, the meter fix crossing time is only within $50 \mathrm{sec}$ of the time computed by the FMS, which does not meet the estimated accuracy requirement of $30 \mathrm{sec}$. The execution error in the airspeed seems to account for half or less of the error in the meter fix crossing time. This part of the error probably cannot be reduced. The other major source of this error is likely error in the descent wind forecast used by the FMS, which Bronsvoort et al. [7] investigated. Stell [8] also found unexplained behavior in the predictions of meter fix crossing time from a B777-200 FMS 


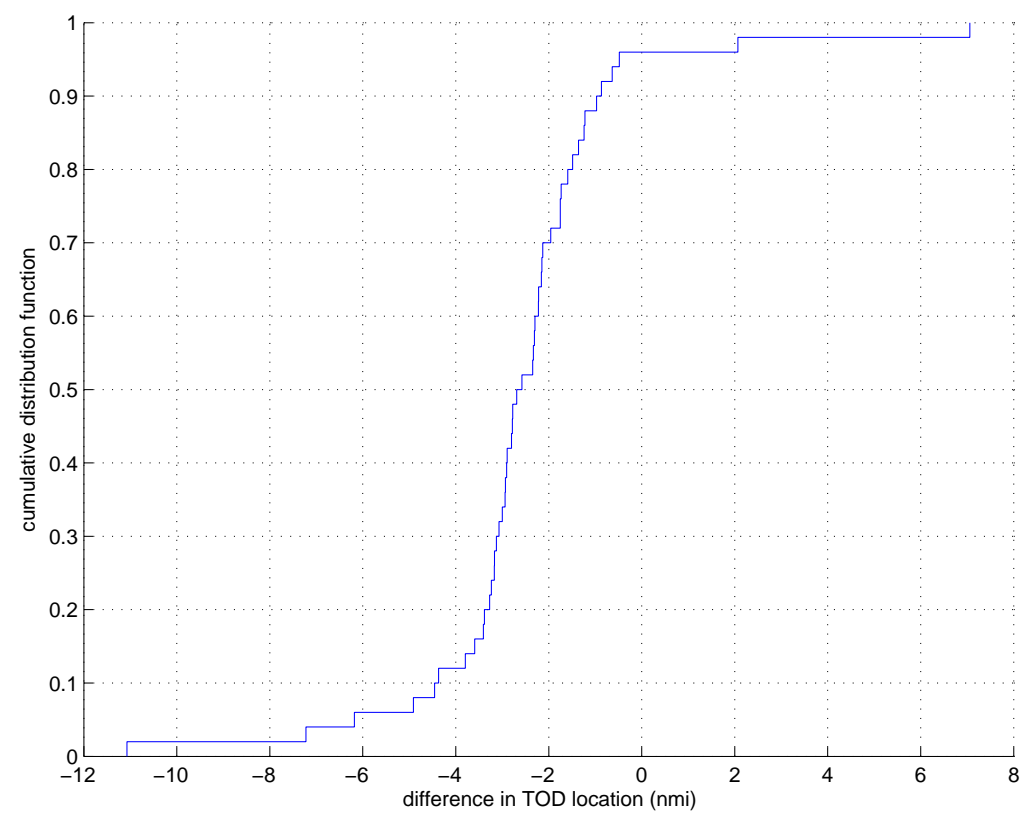

Figure 9. Comparison of Actual and FMS-predicted TOD Location

test bench simulator, which could increase prediction error. Further research is needed to see whether ground predictors might be able to reduce these errors. This might be possible because the autopilot is not directly trying to meet its prediction of the meter fix crossing time but is instead trying to maintain the target descent speed profile.

The TOD location, however, is directly controlled by the autopilot. Therefore, the observed differences between the actual and the intent TOD locations probably reflects unavoidable variation, and a ground predictor is unlikely to be able to predict the TOD location better than the FMS intent trajectory does. This suggests that predicting $95 \%$ of the TOD locations within $5 \mathrm{nmi}$ might be the best achievable. If the FMS intent trajectory is downlinked to ground automation, however, then the conflict detector should take advantage of the bias in these TOD location errors so that it uses a TOD location that has an error of $\pm 2-3 \mathrm{nmi}$. This could greatly assist in separation of arrivals from other arrivals at different cruise altitudes as well as from overflights.

The difference between the actual altitude and the intended altitude depends upon the trajectory constraints used by the FMS. Not surprisingly, specifying the altitude at a point results in significantly smaller errors in the intended altitude.
These results come with a caveat that they are all for one aircraft type operated by two airlines arriving at one airport. Development of a ground predictor and its error models to enable more fuel-efficient descents in congested airspace will require considerably more data for more aircraft types, airlines, and locations under varying weather conditions with various trajectory constraints.

\section{References}

[1] Coppenbarger, Richard A., Rob W. Mead, Douglas N. Sweet, 2007, "Field Evaluation of the Tailored Arrivals Concept for Datalink-enabled Continuous Descent Approach," AIAA 5th Aviation, Technology, Integration, and Operations Conference, Belfast, Northern Ireland.

[2] Stell, Laurel L., 2010, "Predictability of Top of Descent Location for Operational Idle-thrust Descents," AIAA 10th Aviation, Technology, Integration, and Operations Conference, Fort Worth, TX.

[3] Paglione, Mike M., Hollis F. Ryan, 2005, "Comparison of Host Radar Positions to Global Positioning Satellite Positions," 24th Digital Avionics Systems Conference, Washington, D.C.

[4] Stell, Laurel L., 2009, "Flight Management System 
Prediction and Execution of Idle-thrust Descents," 28th Digital Avionics Systems Conference, Orlando, FL.

[5] Klooster, Joel K., Keith D. Wichman, Okko F. Bleeker, 2008, "4D Trajectory and Time-of-arrival Control to Enable Continuous Descent Arrivals," AIAA Guidance, Navigation and Control Conference, Honolulu, HI.

[6] Oprins, Esther, David Zwaaf, Fredrik Eriksson, Koen van de Merwe, Robert Roe, 2009, "Impact of Future Time-based Operations on Situation Awareness of Air Traffic Controllers," 8th USA/Europe Air Traffic Management R\&D Seminar, Napa, CA.
[7] Bronsvoort, Jesper, Greg McDonald, Rodney Potts, Ekkehard Gutt, 2011, "Enhanced Descent Wind Forecast for Aircraft," 9th USA/Europe Air Traffic Management R\&D Seminar, Berlin, Germany.

[8] Stell, Laurel L., 2010, "Analysis of Flight Management System Predictions of Idle-thrust Descents," 29th Digital Avionics Systems Conference, Salt Lake City, UT.

\section{0th Digital Avionics Systems Conference} October 16-20, 2011 